\title{
Wissensvorsprung dank Online First
}

Online publiziert: 7. Februar 2012

(C) Springer-Verlag 2012

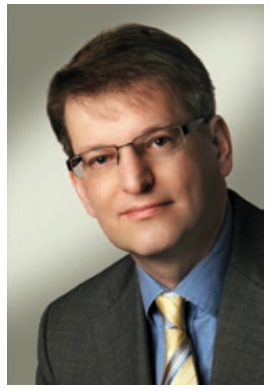

Jörg Klewer

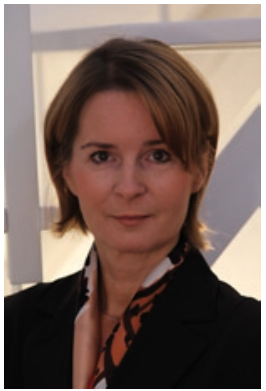

Katja Kupfer-Geißler
Auch wenn HeilberufeSCIENCE schon immer ein online Journal war und nicht gedruckt wurde, bietet Ihnen Springer Medizin nun auch für HeilberufeSCIENCE den Service „Online First“. Damit macht SpringerLink angenommene Beiträge innerhalb von Wochen statt Monaten verfügbar. Mit Online First lesen Sie schon heute, was morgen erst in Form einer neuen Ausgabe erscheint. Online-First-Artikel werden sofort nach ihrer Freigabe durch die Autoren veröffentlicht. Sie sind bereits dann über die so genannte DOI (Digital Object Identifier) such- und zitierbar. So wird elektronische Literatur zur offiziellen Publikation und der Zeitpunkt der Online-Veröffentlichung zum offiziellen Publikationsdatum. Es lohnt sich also, auch „zwischendurch“ mal einen Blick auf SpringerLink zu werfen, um neue Studien aus Pflegemanagement, Pflegepädagogik, Pflegewissenschaften, Gerontologie, Altenpflege oder den Gesundheitswissenschaften zu entdecken.

In der aktuellen Ausgabe von HeilberufeSCIENCE bieten wir Ihnen fünf Originalarbeiten und zwei Kurzmitteilungen. Im ersten Beitrag setzen sich Götz et al. mit den Strukturen der ambulanten Pflegedienste im Bundesland MecklenburgVorpommern auseinander und versuchen eine Einschätzung der zukünftigen Entwicklung des ambulanten Pflegemarktes abzugeben. Aus den Ergebnissen dieser Studie leiten sie außerdem Handlungsempfehlungen für ambulante Pflegedienste und die Pflegepolitik ab. Im nachfolgenden Beitrag untersuchen Schlott und Stummer, welche Anforderungen Angehörige und Führungskräfte der auf Demenzpflege spezialisierten Pflegeheime an einen Heimstandort stellen. In dem anschließenden Beitrag von Hertwig et al. werden die Ergebnisse einer Querschnittstudie präsentiert, bei der in 60 Pflegeheimen in der Stadt Leipzig der telefonische Erstkontakt, das Kunden-Beratungsgespräch sowie die Eindrücke bei einer Vorort-Begehung untersucht wurden. Daran schließt sich die Untersuchung von Meiners et al. an, die sich mit den gerontologischen Aspekten der Anzeigenwerbung bei Deutschlands größtem Lebensmitteldiscounter ALDI auseinander setzt. Die fünfte Originalarbeit von Wache et al. präsentiert die Ergebnisse einer Arbeitszeitanalyse für das Personal in Arztpraxen.

Immer beliebter wird unsere Rubrik der wissenschaftlichen Kurzmitteilung. Sie ermöglicht es Ihnen, interessante Ergebnisse aus Pilotstudien und kleinen Projekten rasch zu publizieren. In der ersten Kurzmitteilung „Golf und Alter in Tirol“" präsentieren Stellwag et al. eine gerontologische Annäherung an das Thema unter demografischem Aspekt. Die Kurzmitteilung von Schaal et al. untersucht den Kenntnisstand von Webdesignern zu Möglichkeiten der barrierefreien Gestaltung von Internet-Auftritten.

Somit möchten wir Sie an dieser Stelle wieder einladen, nicht nur uns Ihre wissenschaftlichen Arbeiten zur Publikation einzureichen, sondern die Artikel und wissenschaftlichen Kurzmitteilungen in dieser Ausgabe von HeilberufeSCIENCE kritisch zu prüfen und zu kommentieren - schreiben Sie uns! Zu guter Letzt möchten wir Sie noch ermuntern, auch einen Blick in die HeilberufeSCIENCE- 
Sonderausgabe mit den Abstracts der Vorträge des Kongresses Pflege 2012 in Berlin zu werfen.

Ihre

$$
\text { 7. Wener kaja kuph-giser }
$$

Prof. Dr. Jörg Klewer - Katja Kupfer-Geißler 